\title{
ARTICLE OPEN \\ A universal, plug-and-play synchronisation scheme for practical quantum networks
}

\author{
Virginia D'Auria ${ }^{1 *}$, Bruno Fedrici ${ }^{1}$, Lutfi Arif Ngah ${ }^{1}$, Florian Kaiser ${ }^{1,2}$, Laurent Labonté ${ }^{1}$, Olivier Alibart ${ }^{1}$ and Sébastien Tanzilli ${ }^{1}$
}

We propose and experimentally demonstrate a plug-and-play, practical, and enabling method allowing to synchronise the building blocks of a quantum network in an all-optical way. Our scheme relies on mature and reliable classical telecommunication and nonlinear optical technologies, and can be implemented in a universal way with off-the-shelf components. Compared to already reported solutions, it allows achieving high-quality synchronisation compatible with high network-operation rate and is free from opto-electronic jitters affecting servo-loop-based configurations. We test our scheme with a genuine quantum optical method in terms of the interference between two photons coming from two remotely synchronised sources spaced by distances of up to $100 \mathrm{~km}$. Measured visibilities well above $90 \%$ confirm the validity of our approach. Due its simplicity and high-quality performance, our scheme paves the way for the synchronisation of long-distance quantum networks based on fibre, free-space, as well as hybrid solutions.

npj Quantum Information (2020)6:21; https://doi.org/10.1038/s41534-020-0245-9

\section{INTRODUCTION}

Quantum networks and synchronisation issues

In the context of digital society, quantum networks promise to combine highly efficient data processing with ultra-secure data exchanges. ${ }^{1-3}$ This vision has motivated the development of crucial constituents including quantum memories, ${ }^{4,5}$ coherent interfaces between different systems, ${ }^{6-9}$ and optical quantum communication links based on quantum teleportation over long fibre connections. ${ }^{10-13}$ Nevertheless, the development of operational quantum networks remains hindered by the lack of practical synchronisation methods that allow the different building blocks to work together under high timing accuracy. If different synchronisation strategies have been investigated both in pulsed and in continuous wave regimes, they suffer from important limitations in terms of achievable operation rates and distances. ${ }^{14-}$ 17 The pulsed regime offers the advantage of introducing an intrinsic time-binning. However, in most of reported realisations, synchronisation has demanded the implementation of sophisticated, and hardly scalable, phase-locked loops ${ }^{15,18}$ or atomic clocks and dedicated electronics. ${ }^{19,20}$ As relevant and recent examples, two out-of-the-laboratory demonstrations ${ }^{11,12}$ have established the state-of-the-art for discrete variable quantum networks, by showing entanglement teleportation across metropolitan areas. In both schemes, the synchronisation of the network nodes relies on custom feedback systems. These latter, as for all strategies relying on servo-loops, ${ }^{15-18}$ are based on optoelectronic conversions that come at the price of unavoidable timing-jitters. To mitigate their effect, photons carrying the quantum information must be filtered so as to have long coherence times and relax the constraints on the synchronisation. This requires the use of spectral filtering stages ${ }^{16}$ and, more importantly, poses a fundamental limitation to the maximum clocking regime in order to avoid signals from different photons to superpose. Alternatively, a different approach is based on continuous operation regime and on the post-selection of single time mode events by means of detection. ${ }^{16,21}$ In this case, as no intrinsic time-binning is provided by the continuous lasers, detector timing jitters become the principal source of time uncertainty. However, to comply with typical detection jitters (at best few tens of ps), ${ }^{22}$ extremely narrow spectral filtering stages are demanded with a dramatic effect on working rate.

In this study, we consider the pulsed regime and show that an efficient, universal, and plug-and-play solution to the quantum network synchronisation is provided by the combination of offthe-shelf classical telecommunication and nonlinear optics technologies. Our idea is to exploit classical telecom know-how to distribute an all-optical clock signal over a fibre network and to apply locally adapted nonlinear optical stages ${ }^{17}$ to tailor its spectral properties and make it drive directly the devices located at the different nodes. By using suitable nonlinear optical conversions, this scheme can be employed to deliver a common time-reference to distant quantum systems with no a priori restrictions on either their nature or number. We successfully tested it by synchronising two distant photon pair sources. Compared to other reported solutions, our fully optical method offers high synchronisation quality without the need for complex clock or control systems. This strategy dramatically reduces the experimental overhead and bypasses accuracy limitations due to finite speed ${ }^{18}$ and timing-jitters of current servo-loop systems. ${ }^{23}$ Its extreme simplicity, universality, and feasibility with current technologies make our synchronisation scheme a valuable strategy for future, long distance, quantum networks made with fibres and/or in-space architectures.

\section{A timing-jitter-free solution}

In a practical situation, the master clock is represented by a pulsed laser emitting at a telecom wavelength compatible with long distance distribution in optical fibres. Classical telecom technology represents a particularly convenient work frame by providing ultra-fast optical clocks with pulse repetition rates up to $10 \mathrm{GHz}^{24}$ chromatic dispersion compensation modules, high gain erbium-doped fibre amplifiers (EDFA), and low loss

${ }^{1}$ Université Côte d'Azur, CNRS, Institut de Physique de Nice (INPHYNI), UMR 7010, Parc Valrose, Nice Cedex-2, 06108, France; ${ }^{2}$ Present address: The 3rd Institute of Physics, University of Stuttgart and Institute for Quantum Science and Technology IQST, Germanyfaffenwaldring 57, 70569 Stuttgart, Germany. *email: Virginia.DAuria@univ-cotedazur.fr 


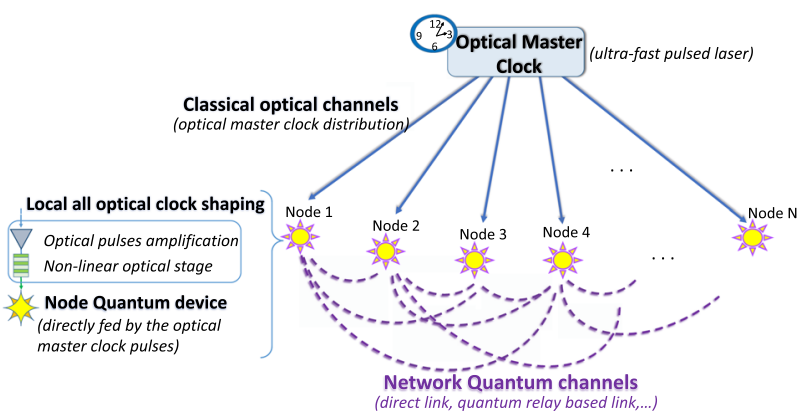

Figure 1 Conceptual architecture of our synchronisation procedure based on an all-optical master clock. Optical clock laser pulses are distributed in parallel to different nodes of the quantum network via classical optical channels. At each of the nodes, depending on the specific situation, the clock pulses are locally shaped in an all-optical fashion via amplification and nonlinear optical stages and used to feed directly the quantum devices (entanglement generation or storage) present at the node. By doing so, the nodes driven by the same optical clock are automatically synchronised without the introduction of any jitters and without the need for control systems. As for general quantum networks, they can now be connected by quantum communication channels and used to share quantum information.

fibre components, including dense wavelength demultiplexing stages (DWDM). ${ }^{25}$

As shown in Fig. 1, master clock pulses are distributed in parallel to different network nodes and are locally used to optically pump remote quantum devices. By doing so, the quantum systems (nodes 1 to $n$ in Fig. 1) are all driven by synchronous optical pulses and are, as a consequence, automatically provided with the same intrinsic timing reference. We stress that, since the clock laser does not convey any quantum information, its routing over the network can fully benefit from standard classical telecommunication tools. Undesired nonlinear effects caused by the propagation of ultrashort optical pulses over long optical fibres ${ }^{26}$ can be overcome by using Fourier-transform-limited optical pulses lasting a few picoseconds (instead of femtoseconds). At the same time, advances in nonlinear optics ensure that off-the-shelf components can be employed, if necessary, to efficiently shape the master clock signal in the frequency domain so as to make it compatible with a specific and operational quantum device. ${ }^{17}$ This includes the possibility, after the distribution, of obtaining pulses that are longer than picosecond by means of adequate local spectral filtering.

Conceptually, the entire process of clock sharing, and manipulation is all-optical and free from parasite timing-jitters, typically in the order of tens of $\mathrm{ps}^{14,23}$ that arise from opto-electronic conversions in servo-loops. In this regard, we emphasise that random timing-jitters are responsible for the main limitation in timing precision for most of the reported synchronisation configurations. Moreover, they represent an intrinsic limit to maximum achievable clock rates as they cause signals from different clock cycles to superpose. ${ }^{22}$ In our scheme, the timing accuracy is in principle only defined by the stability of the master clock repetition rate, whose uncertainty is well below $100 \mathrm{fs}$ for off-the-shelf ultra-fast lasers and can go down to a fraction of a femtosecond in research-grade systems. ${ }^{27}$ Accordingly, our method guarantees high precision and is straightforwardly compatible with ultra-fast operation driven by master clock lasers paced at $\mathrm{GHz}$ repetition rates. ${ }^{24}$ Eventually, in the particular case of quantum network configurations requiring an interferometric phase stabilisation, ${ }^{28}$ our method is fully compliant with the addition of an optical frequency reference, as achieved in large infrastructures for time-frequency dissemination. ${ }^{29}$

\section{RESULTS AND DISCUSSION}

Synchronisation tests on short distances

To demonstrate the validity of our strategy, we apply it to the synchronisation of two remote photon pair sources (PPSs) in a quantum relay configuration (see Fig. 2). Such an architecture lies at the heart of reliable quantum networks, because synchronous and independent entangled photon sources and relay nodes are key elements to any quantum teleportation-based optical quantum link. ${ }^{10,12}$ In our study, the all-optical synchronisation is provided by a commercial ultra-fast optical clock (Pritel-UOC) emitting 2 ps long pulses at $1540 \mathrm{~nm}$ with a timing jitter $\leq 100 \mathrm{fs}$. In order to obtain a quantitative measurement of the PPS synchronisation, we use the genuine quantum optical method referred to as Hong Ou Mandel (HOM) distinguishability test between independent photons coming each from one of the two sources. ${ }^{23}$ The interference visibility provides the relevant witness of the synchronisation quality. A perfect photon coalescence, i.e. a visibility of $100 \%$, corresponds to two interfering photons indistinguishable in the temporal modes in which they are emitted and synchronised with an accuracy better than their coherence time, namely, their intrinsic time uncertainty. ${ }^{23}$ Conversely, any timing-jitter accumulated during the photon generation process or through the quantum channel would induce a random fluctuation around the dip minimum and would result in decreased visibility. We qualify our synchronisation scheme by considering different separations between the two PPSs, including asymmetric configurations where, as in real-world configurations, the distances between the clock laser and each of the PPS are unbalanced. Due to the importance of the master clock laser characteristics for the entire setup, we start by focusing only on the relevance of its coherence properties on the synchronisation quality and temporarily put aside supplementary difficulties linked to long-distance operation. By means of preliminary classical interference (not represented), we first measured the master clock coherence length to be of $L_{c} \approx 200 \mathrm{~m}$. Subsequently, we perform Hong-Ou-Mandel (HOM) experiments by considering the case of a symmetric configuration in which the master clock laser is set perfectly halfway between the two sources, spaced $\approx 10$ and that of an asymmetric one in which the distance between the clock laser and each of the PPS shows a distance mismatch of $\Delta L \approx 400 \mathrm{~m}>L_{\mathrm{c}}$. Figure $3 \mathrm{a}, \mathrm{b}$ report the HOM dips that were measured in the two operation conditions. As can be seen, there is no difference between the two experimentally obtained results and, in both configurations, the HOM profile is recovered with an extremely high raw visibility of $100 \%$. As accidental four-fold coincidences between dark counts, or dark counts and single photons, are negligible, this measured raw HOM visibility coincides with its net value corrected for detection noise. ${ }^{23}$ The excellent result on visibility conceptually confirms the high quality of our synchronisation protocol and, at the same time, validates the lack of possible constraints related to the laser coherence or its distance with respect to the sources.

As a final remark, we observe that by means of error propagation, we can estimate, from our experimental data, a lower bound to HOM visibility of $95.5 \%$. Under the hypothesis of Gaussian distributed timing uncertainties, this value sets our minimum detectable jitters to $3 \mathrm{ps}$. A better accuracy would be obtained with a richer statistic of four-fold events. Without changing the pumping conditions, and, as a consequence, the photon-pair emission statistics, this can be done by using a clock with a higher repetition rate of $10 \mathrm{GHz}$ and state-of-the-art superconducting detector with ultra-low detection jitters and quantum efficiency close to $90 \% .^{30}$ This would increase the overall count by a factor 40 and lead to a measured jitter $\sim 0.5$ ps. 


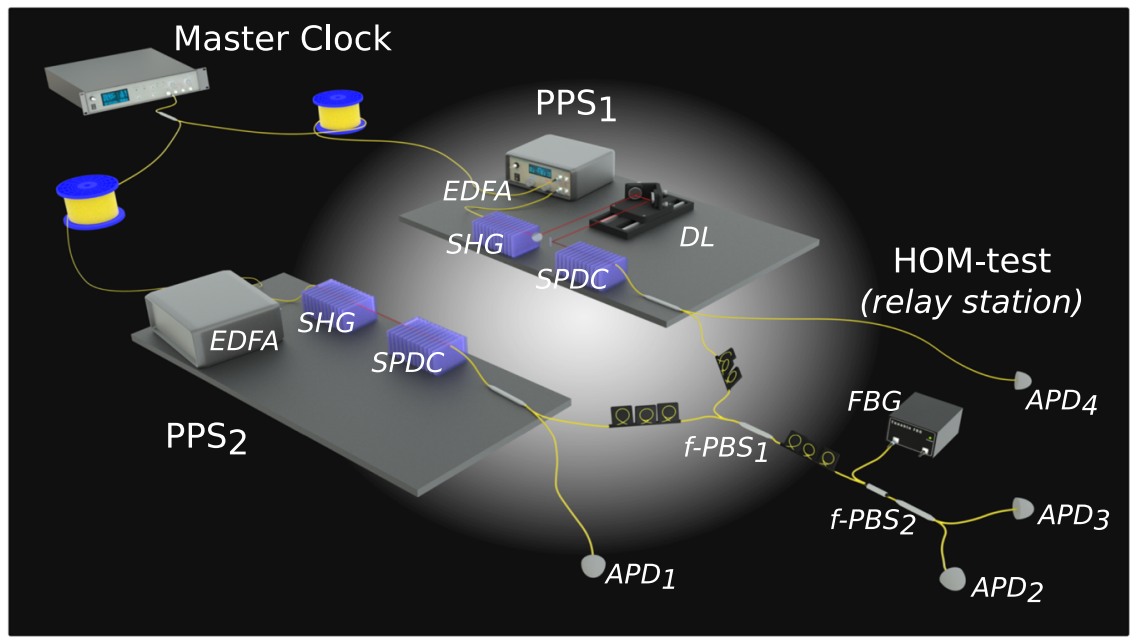

Figure 2 Experimental setup employed for testing the synchronisation of two remote photon pair sources (PPSs). Conceptually, this setup is similar to those used in entanglement teleportation experiments. Optical pulses at $1540 \mathrm{~nm}$ from the all-optical master clock are distributed via optical fibres to two PPSs. At the PPS nodes, pulses are locally amplified (EDFA), frequency doubled (SHG) and used to pump the photon pair generation stages (SPDC). The SPDC and the SHG stages are packaged into in-house boxes so as to reduce thermal fluctuations and their temperature is actively stabilised. At the output of the SPDC, the paired photons are separated as a function of their frequency by a fibre wavelength demultiplexer (WDM) and the source synchronisation is qualified via a HOM experiment between the photons at $1536.27 \mathrm{~nm}$ heralded by their twins at $1543.73 \mathrm{~nm}$. In order to obtain the HOM dip, a delay line at PPS ${ }_{1}$ allows changing, in a controlled way, the arrival time of one of the two interfering photon at the relay station. Detector $A P D_{2}$ corresponds to four free-running avalanche photo-diode (IDQ 220) whose outputs are connected to a logic OR gate. They each feature a quantum detection efficiency of $25 \%, 7 \mu$ s dead time, and a dark count probability of $10^{-6} / \mathrm{ns}$. APD 2 provides a trigger signal to the three other APDs (IDQ 210 ) operated in a gated mode and all showing $20 \%$ quantum detection efficiency, $7 \mu \mathrm{s}$ dead time, and a dark count probability of $10^{-5} / \mathrm{ns}$. APD's saturation rate is $\approx 60 \mathrm{kHz}$ for all individual APDs and $\lesssim 200 \mathrm{kHz}$ for the multiplexed detector. These values impose a limitation to the mean number of photon pairs that are generated by each source and, in turns, to the maximum pump power at the SDPC input. Typically employed pump power at $770 \mathrm{~nm}$ are $\sim 15 \mathrm{~mW}$.

Long distance operation

Subsequently, in order to check the validity of our method for practical network purposes, we perform the synchronisation of two PPSs spaced by $100 \mathrm{~km}$, i.e. each at $50 \mathrm{~km} \pm 1 \mathrm{~km}$ from the master clock location. The tests are effectuated in-the-laboratory, by employing $50 \mathrm{~km}$ long spools of optical fibres. Nevertheless, this configuration reproduces a realistic long-distance operation, where the distribution of the maser-clock pulses over optical fibres is affected by strong propagation losses and non-negligible chromatic dispersion effects. Moreover, such a protocol must cope with thermal fluctuations in the long fibre links.

In our realisation, the clock pulses directed toward each source have a mean power of $\sim 1.25 \mathrm{~mW}$. This optical power is attenuated by propagation losses in the fibres $(-10 \mathrm{~dB})$ and extra-losses at the chromatic dispersion modules $(-6 \mathrm{~dB})$, thus leading to a mean power of $25 \mu \mathrm{W}$. At the same time, due to chromatic dispersion, the duration of the clock pulses broadens from 2 ps to $0.85 \mathrm{~ns}$. As already stated, to face these effects, our scheme fully benefits from standard telecom technology and both attenuation and chromatic dispersion are efficiently managed thanks to plug-andplay EDFA and chromatic dispersion compensation modules based on adapted dispersion-shifted fibres. ${ }^{25}$ Accordingly, the most critical point lies in the fibre refractive index temperature dependence $^{31}$ that can modify the optical distances between the two sources. For our experimental setup, thermal variations result in a drift of the HOM dip position of up to $5 \mathrm{~mm} / \mathrm{h}$. This figure has to be compared with a dip full-width-half-maximum of $\approx 6 \mathrm{~mm}$ and with typical measurement integration time of $1 \mathrm{~h} /$ point. This effect can seriously compromise the synchronisation quality and directly maps to a shift of the HOM dip position in real time. Nevertheless, we stress that, compared to fast and random noise due to optoelectronic time-jitters, thermal fluctuations induce slow variations, an issue that has been comprehensively addressed in the past and which can be easily corrected with a standard-performance fibre-length tracking system. ${ }^{32}$

In order to implement an active length stabilisation system, we use the fibre spools that connect the two PPSs to the master clock as the two arms of a classical Mach-Zehnder interferometer and employ a fraction of the intensity of the master clock laser to track the position of the classical interference fringes.

The system corrects length fluctuations with an accuracy of $\sim 0.3 \mathrm{~mm}$, to be compared with a dip full-width-at-half-maximum of $\approx 6 \mathrm{~mm}$. We stress that this is not the state-of-the-art value for the stabilisation of long distance interferometers and higher accuracies are reported in the literature. ${ }^{10,12,32}$ As shown in Fig. 3c, the experimental results obtained with our correction system show a high raw visibility above $90.5 \%$, therefore validating our synchronisation approach for long distance operation. Note that this visibility value is compatible with our in-house servo-loop characteristics, and its low accuracy. Visibilities $>95 \%$ would be straightforwardly achieved, with a more accurate correction system.

\section{CONCLUSION}

In conclusion, we have presented and experimentally demonstrated a novel protocol that solely and simply relies on an alloptical master clock laser, the timing precision of which guarantees the synchronisation of a network blocks over long distance. Our concept exploits elementary off-the-shelf telecom technologies and optical frequency conversion stages to enable universal plug-and-play optical synchronisation of any quantum node. The synchronisation approach proposed here is extremely versatile and allows in principle to add to a quantum network as many nodes (quantum memories, sources, etc.) as necessary and this whatever their nature is. In this sense, our synchronisation method can therefore be seen as an enabling method for scaling 
Optical delay (ps)

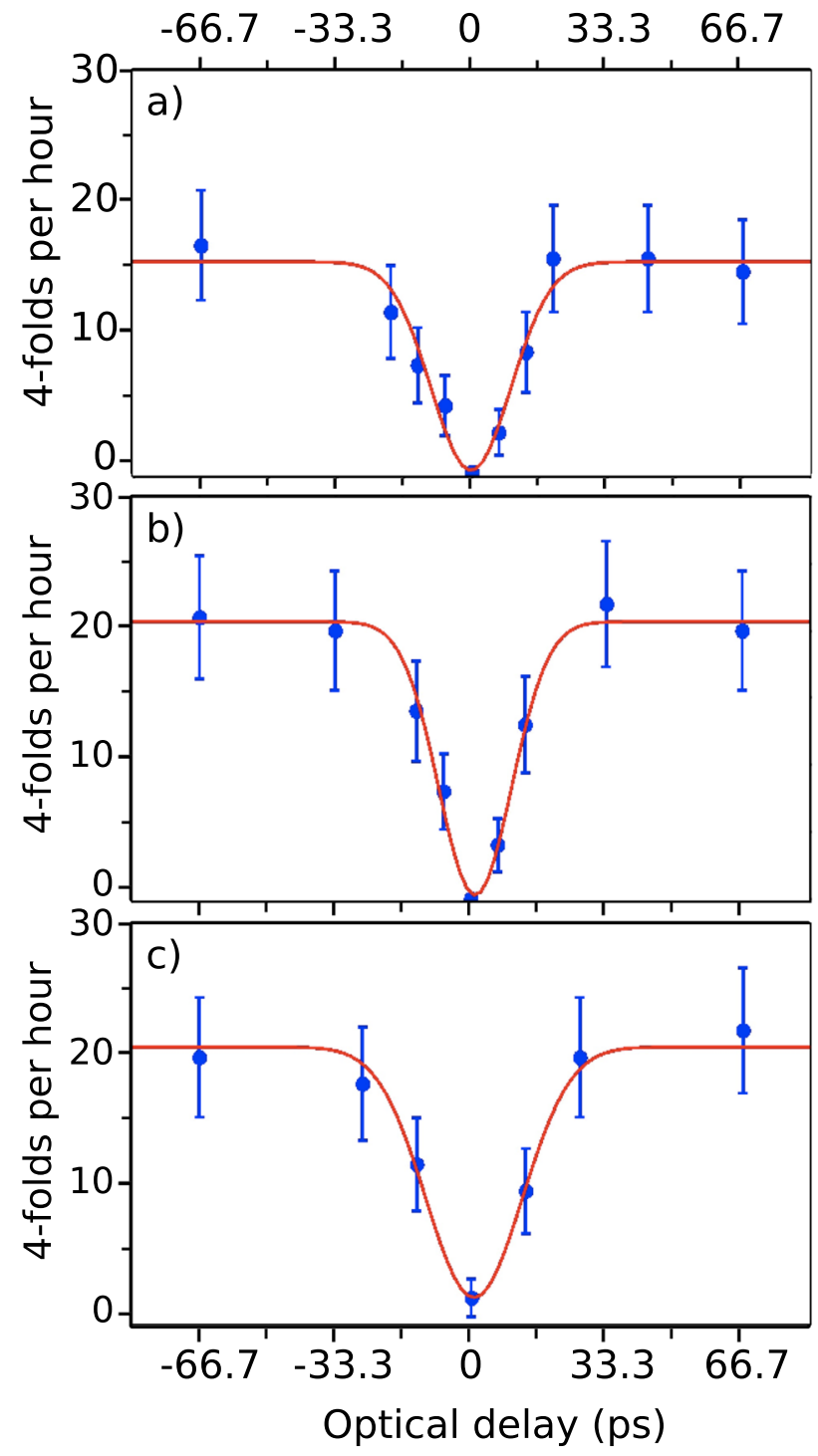

Figure 3 Raw experimental results of the HOM interference between photons emitted by PPS $_{1}$ and PPS $_{2}$. The curves show four-fold coincidences between the two heralding detectors and the two detectors at the relay station. Error bars are given as the standard deviation of Poissonian distributions. a HOM dip for the case of two PPSs spaced by $10 \mathrm{~m}$ with the master clock put perfectly halfway; b HOM dip for an asymmetric configuration with a length mismatch $\Delta L \approx 400 \mathrm{~m} \geq L_{c}$; c HOM dip for the case of two PPSs spaced by $100 \mathrm{~km}$ with the master clock at a distance of $\sim 50 \pm 1 \mathrm{~km}$ from each source. Red lines represent Gaussian fits on the experimental data. We underline higher four-fold coincidence rates could be achieved by simply replacing the APDs by superconducting single photon detectors exhibiting high saturation rates in the $\mathrm{MHz}$ regime and detection efficiencies up to $90 \%{ }^{18,27}$ and thus allowing to operate the experiment at higher rates.

up quantum networks. Compared to previously reported methods, we emphasise that our solution dramatically reduces the overhead for experimental resources. We validate our strategy for the synchronisation of distant photon pair sources based on the SPDC process by observing extremely high-quality two-photon interference over $100 \mathrm{~km}$. We stress that, provided suitable nonlinear optical stages are available, our strategy is compatible with generic optical devices at the nodes of quantum networks, thus providing a relevant tool for future practical architectures.

\section{MATERIALS AND METHODS}

A quantum relay configuration

To demonstrate the validity of our strategy, we apply it to the synchronisation of two remote PPSs in a quantum relay configuration (see Fig. 2). The all-optical synchronisation is provided by a commercial ultra-fast optical clock (Pritel-UOC) emitting 2 ps long pulses at $1540 \mathrm{~nm}$, corresponding to the standard channel 47 of the International Telecommunication Union (ITU) wavelength grid. Typical mean powers are $\sim 2.5 \mathrm{~mW}$. The laser works at repetition rate of $2.5 \mathrm{GHz}$ with a residual timing error on the pulses' emission times $\leq 100 \mathrm{fs}$. Its output is split into two at a 50:50 fibre beam splitter and distributed via standard optical fibres to the two remote PPSs. The PPSs generate paired photons at telecom wavelengths via type-0 SPDC in in-house periodically poled lithium niobate waveguides. ${ }^{17,23,24}$ Accordingly, at each PPS station, master clock pulses are amplified up to $1 \mathrm{~W}$ using EDFA and frequency converted to $770 \mathrm{~nm}$ via second harmonic generation (SHG) as required to use them to pump the SPDC process. The source synchronisation is guaranteed only if the optical pulses pumping the remote generation processes do not exhibit relative time-jitters. This condition is automatically satisfied when the SPDC stages are directly pumped by the same master clock laser. ${ }^{23}$ Typical pump powers before the SPDC stages are set to $\sim 15 \mathrm{~mW}$ at $770 \mathrm{~nm}$ so as to avoid saturation at the detection stages. Correspondingly, emitted photon pairs follow a Poissonian statistics ${ }^{17}$ with mean number of pairs per pump pulse $\sim 0.0012$. We note that, in view of long distance applications, time-bin-entangled photons can be conveniently obtained at the output of type 0 SPDC, by locally adding on the path of pump pulses at $770 \mathrm{~nm}$ in an unbalanced interferometer. ${ }^{14,17}$ Alternatively, polarisation entanglement can be obtained by replacing our non-linear waveguides with different ones allowing for a type II phase-matched SPDC. ${ }^{14}$

Following a typical teleportation scheme, signal photons at $1543.73 \mathrm{~nm}$ (ITU channel 42) coming from the PPSs are routed by a fibre wavelength de-multiplexing stage (WDM) ${ }^{17,23}$ towards distant communication partners to be locally detected by two avalanche photodiodes $\left(A P D_{1}\right.$ and $A P D_{4}$ in Fig. 2). Idler photons at $1536.27 \mathrm{~nm}$ (ITU channel 50) are directed towards a central relay station where their synchronisation is evaluated (relay station in the figure). Signal and idler photons are filtered so as to have a spectral bandwidth of $800 \mathrm{~nm}(100 \mathrm{GHz})$ and $200 \mathrm{~nm}(25 \mathrm{GHz})$, corresponding to coherence times of 4 and $17 \mathrm{ps}$, respectively. Note that, in the context of quantum communication over long distances, the ps-regime has been demonstrated to enable near perfect two-photon interference while being fully compatible with standard telecom components and optical filters. ${ }^{23}$

To evalutate the quality of the synchronisation, we use HOM distinguishability test between independent photons coming each from one of the two sources. ${ }^{33}$ The visibility of the associated two-photon interference pattern represents a pertinent figure-of-merit assessing the quality of the synchronisation. To this end, we follow the very standard approach of quantum relays ${ }^{1,12,23}$ and make both our PPSs work as heralded single photon sources. In principle, this strategy allows optimising the performances of the HOM test in terms of two-photon interference visibility. In our experiment, detection signals of photons at $1543.73 \mathrm{~nm}$ at $A P D_{1}$ and $\mathrm{APD}_{4}$. announce the presence of heralded idler photons at $1536.27 \mathrm{~nm}$ from EPPS 1 and EPPS 2 , respectively. ${ }^{24}$

\section{A robust HOM setup}

The HOM test setup interference employed in our experiment relies on polarisation-based two-photon interference. ${ }^{12}$ The heralded photons from the two sources are sent to a fibre polarisation beam splitter (f-PBS), which cleans their respective polarisations and projects their original state on the quantum state $|\psi\rangle=|H\rangle|V\rangle$. A polarisation controller (PC) is used to rotate the polarisation state of the pair by $45^{\circ}$, leading to the state: $|\psi\rangle_{\mathrm{PC}}=\frac{1}{2}(|H\rangle|V\rangle+|V\rangle|V\rangle-|H\rangle|H\rangle-|V\rangle|H\rangle)$. The PC output is subsequently sent to a second f-PBS whose outputs are each connected to an $\mathrm{APD}\left(\mathrm{APD}_{2}\right.$ and $\left.A \mathrm{AD}_{3}\right)$. The relative photon arrival times are changed by a dedicated optical delay-line (DL) and the coincidences between the counts of four APDs are recoded as a function of the relative delay. If the photons are perfectly indistinguishable in all degrees of freedom, namely spatial, spectral and temporal modes, except for their polarisation upon impinging the first f-PBS, quantum theory predicts that contributions $|H\rangle|V\rangle$ and $|V\rangle|H\rangle$ interfere destructively. As a consequence, when the photon delay is set to zero and a high-quality and stable source synchronisation is achieved, the quantum state after the action of both the f-PBS leads to the state $\cdot|\psi\rangle_{\text {HOM }}=\frac{1}{\sqrt{2}}(.|V\rangle \cdot|V\rangle-.|H\rangle \cdot|H\rangle)$ and detectors $\mathrm{APD}_{2}$ and $\mathrm{APD}_{3}$ can 


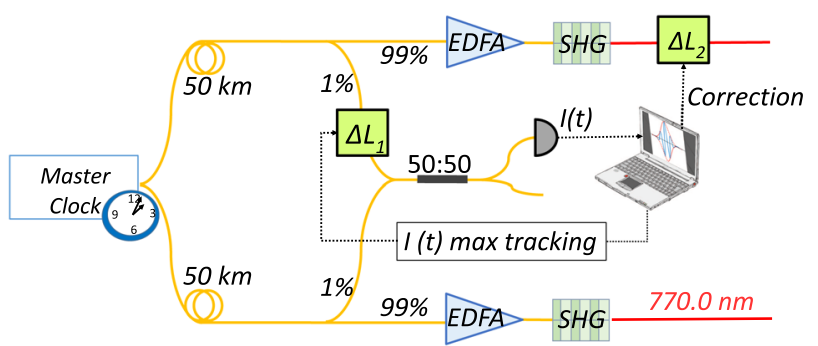

Figure 4 Fibre length stabilisation setup for the HOM test in the case of two PPS spaced by $\mathbf{1 0 0} \mathbf{~ k m}$. The delay line $\Delta \mathrm{L}_{1}$ allows us to check the maximum of the Mach-Zehnder classical interference fringes. In case of unwanted shift, a correction signal is sent to a second delay line, $\Delta \mathrm{L}_{2}$ in order to conveniently adjust the HOM dip scan. The stabilisation system is in-house with off-the-shelf and lowcost standard electronic components.

never fire together. A dip in the four-fold coincidences among detection signals from all the APDs is thus observed.

The interference visibility provides the relevant witness of the synchronisation quality. We recall that the success of any teleportation protocol critically depends on two-photon interference, ${ }^{16,24}$ thus making the exploitation of a HOM-type interferometers an extremely pertinent testbed to verify the practical capacity of any synchronisation technique. At the same time, compared with the original HOM scheme where single photons are mixed on a 50:50 beam-splitter, as discussed in ref. ${ }^{34}$, our chosen configuration offers two main advantages: (a) as the first f-PBS acts as a polarisation filter, any polarising rotation of the impinging photons only decreases the rate of detected counts but does not affect the interference visibility; (b) spectral filtering of signal photons originating from separate SPDC sources is performed by a single fibre Bragg grating (FBG) filter, placed immediately after the first f-PBS. Accordingly, the indistinguishability in terms of spectral mode is automatically ensured and it is immune to the shifting wavelength of the FBG filter. Note that the bandwidth of photons at $1536.27 \mathrm{~nm}(25 \mathrm{GHz})$ has been chosen so as to have pure heralded single photon states, despite parasitic frequency correlations due to the SPDC process. ${ }^{23,24}$ For the test over long distances, in order to implement an active length stabilisation system, we use the fibre spools that connect the two PPSs to the master clock as the two arms of a classical Mach-Zehnder interferometer and employ a fraction of the intensity of the master clock laser to track the position of the classical interference fringes (see Fig. 4). We correct optical length variations dynamically by adjusting the optical delay line used for the HOM interference in response to the variations. We note that in our experiment, the Mach-Zehnder output beam coupler combines optical signals immediately before the spontaneous parametric down conversion (SPDC) stages. In a real-world configuration the output beam splitter can be, for instance, set at the relay station, so as to take into account also length fluctuations and polarisation dispersion in the fibres downstream from the PPSs. Other configurations can rely on Michelson-like interferometers, where a fraction of the optical clock counter-propagates back and forth through each of the spools and provides information on their optical length.

\section{DATA AVAILABILITY}

The data that support the findings of this study are available from the corresponding author upon reasonable request.

Received: 7 October 2019; Accepted: 5 January 2020; Published online: 10 February 2020

\section{REFERENCES}

1. Kimble, H. J. The quantum internet. Nature 453, 1023 (2008).

2. Simon, C. Towards a global quantum network. Nat. Photonics 11, 678 (2017).

3. Wehner, S., Elkouss, D. \& Hanson, R. Quantum internet: a vision for the road ahead. Science 362, eaam9288 (2018).

4. Saglamyurek, E. et al. Quantum storage of entangled telecom-wavelength photons in an erbium-doped optical fibre. Nat. Photonics 9, 83 (2015).
5. Parigi, V. et al. Storage and retrieval of vector beams of light in a multiple-degreeof-freedom quantum memory. Nat. Commun. 6, 7706 (2015).

6. Woerkom, D. Jvan et al. Microwave photon-mediated interactions between semiconductor qubits. Phys. Rev. X 8, 041018 (2018).

7. Maring, N. et al. Photonic quantum state transfer between a cold atomic gas and a crystal. Nature 551, 485 (2017).

8. Kaiser, F. et al. Toward continuous-wave regime teleportation for light matter quantum relay stations. IEEE J. Sel. Top. Quantum Electron. 21, 69 (2015).

9. Kaiser, F. et al. Quantum optical frequency up-conversion for polarisation entangled qubits: towards interconnected quantum information devices. Opt. Express 27, 25603 (2019).

10. Sun, Q. et al. Entanglement swapping over $100 \mathrm{~km}$ optical fiber with independent entangled photon-pair sources. Optica 4, 1214 (2017).

11. Sun, Q.-C. et al. Quantum teleportation with independent sources and prior entanglement distribution over a network. Nat. Photonics 10, 671 (2016).

12. Valivarthi, R. et al. Quantum teleportation across a metropolitan fibre network. Nat. Photonics 10, 676-680 (2016).

13. Jin, R.-B., Takeoka, M., Takagi, U., Shimizu, R. \& Sasaki, M. Highly efficient entanglement swapping and teleportation at telecom wavelength. Sci. Rep. 5, 9333 (2015).

14. Xia, X.-X., Sun, Q.-C., Zhang, Q. \& Pan, J.-W. Long distance quantum teleportation. Quantum Sci. Technol. 3, 014012 (2018).

15. Tao, Y. et al. Experimental synchronization of independent entangled photon sources. Phys. Rev. Lett. 96, 110501 (2006).

16. Halder, M. et al. Entangling independent photons by time measurement. Nat. Phys. 3, 692-695 (2007)

17. Alibart, O. et al. Quantum photonics at telecom wavelengths based on lithium niobate waveguides. J. Opt. 18, 104001 (2016).

18. Kaltenbaek, R., Prevedel, R., Aspelmeyer, M. \& Zeilinger, A. High-fidelity entanglement swapping with fully independent sources. Phys. Rev. A 79, 040302 (2009).

19. Shelton, R. K. et al. Phase-coherent optical pulse synthesis from separate femtosecond lasers. Science 293, 1286 (2001).

20. Cundiff, S., Kolner, B., Corkum, P., Diddams, S. \& Telle, H. Introduction to the issue on stabilization of mode-locked lasers. IEEE J. Sel. Top. Quantum Electron. 9, 969 (2003).

21. Halder, M. et al. High coherence photon pair source for quantum communication. New J. Phys. 10, 023027 (2008).

22. Hadfield, R. H. Single-photon detectors for optical quantum information applications. Nat. Photonics 3, 696 (2009).

23. Aboussouan, P., Alibart, O., Ostrowsky, D. B., Baldi, P. \& Tanzilli, S. High-visibility two-photon interference at a telecom wavelength using picosecond-regime separated sources. Phys. Rev. A 81, 021801 (2010).

24. Ngah, L. A., Alibart, O., Labonté, L., D'Auria, V. \& Tanzilli, S. Ultra-fast heralded single photon source based on telecom technology. Laser Photon. Rev. 9, L1-L5 (2015).

25. Aktas, D. et al. Entanglement distribution over $150 \mathrm{~km}$ in wavelength division multiplexed channels for quantum cryptography. Laser Photon. Rev. 10, 451 (2016).

26. Agrawal, G. P. Nonlinear Fiber Optics. (Academic Press, Boston, 2006).

27. Benedick, A. J., Fujimoto, J. G. \& Kärtner, F. X. Optical flywheels with attosecond jitter. Nat. Photonics 6, 97 (2012).

28. Rozpedek, F. et al. Near-term quantum-repeater experiments with nitrogenvacancy centers: overcoming the limitations of direct transmission. Phys. Rev. A 99, 052330 (2019).

29. Lisdat, C. et al. A clock network for geodesy and fundamental science. Nat. Commun. 7, 12443 EP (2016).

30. Miki, S., Yamashita, T., Terai, H. \& Wang, Z. High performance fiber-coupled NbTiN superconducting nanowire single photon detectors with Gifford-McMahon cryocooler. Opt. Express 21, (2013).

31. Leviton, D. B. \& Frey, B. J. Temperature-dependent absolute refractive index measurements of synthetic fused silica. Proc. SPIE 6273, Optomechanical Technologies for Astronomy, 62732K (2006).

32. Minář, J., Riedmatten, H., de Simon, C., Zbinden, H. \& Gisin, N. Phase-noise measurements in long-fiber interferometers for quantum-repeater applications. Phys. Rev. A 77, 052325 (2008).

33. Loudon, R. The Quantum Theory of Light (Oxford University Press, 2000).

34. Kaiser, F. et al. High-quality polarization entanglement state preparation and manipulation in standard telecommunication channels. New J. Phys. 14, 085015 (2012).

\section{ACKNOWLEDGEMENTS}

This work has been conducted within the framework of the OPTIMAL project, funded by the European Union by means of the Fond Européen de développement regional (FEDER). The authors acknowledge financial support from Agence Nationale de la 
Recherche (ANR) through the Conneqt (ANR-2011-EMMA-0002), Hylight (ANR-17CE30-0006-01) and SPOCQ (ANR-14-CE32-0019) projects, Conseil Régional PACA through the Distance project (Apex 2011: 2001-06938), CNRS through Conneqt (PEPS INSIS 2011), the Majlis Amanah Rakyat (MARA), Université Côte d'Azur (SPEED, CSI 2015) and the French government through its Investments for the Future programme under the Université Côte d'Azur UCA-JEDI project (Quantum@UCA) managed by the ANR (ANR-15-IDEX-01). The authors acknowledge technical support from DRAKA (Prysmian group) and IDQuantique. The illustrated synchronisation scheme has been secured by an international patent (CNRS patent no. FR11/58857 du 30/09/2011 already delivered in Europe, USA and Japan, pending in China). Authors would like to thank Anthony Martin for stimulating discussions and Fabien Kéfélian for the analysis of stability of our master clock laser.

\section{AUTHOR CONTRIBUTIONS}

V.D. has led the project since its conception and supervised all experiments. B.F. has conducted most of the experimental work including the HOM measurement. L.A.N. and L.L. have worked to the nonlinear optical stage optimisation, and F.K. and O.A. on the detection and stabilisation of the experimental setup. S.T. conceived the synchronisation scheme and co-supervised the project with V.D.

\section{COMPETING INTERESTS}

The authors declare no competing interests.

\section{ADDITIONAL INFORMATION}

Correspondence and requests for materials should be addressed to V.D.

Reprints and permission information is available at http://www.nature.com/ reprints

Publisher's note Springer Nature remains neutral with regard to jurisdictional claims in published maps and institutional affiliations.

Open Access This article is licensed under a Creative Commons Attribution 4.0 International License, which permits use, sharing, adaptation, distribution and reproduction in any medium or format, as long as you give appropriate credit to the original author(s) and the source, provide a link to the Creative Commons license, and indicate if changes were made. The images or other third party material in this article are included in the article's Creative Commons license, unless indicated otherwise in a credit line to the material. If material is not included in the article's Creative Commons license and your intended use is not permitted by statutory regulation or exceeds the permitted use, you will need to obtain permission directly from the copyright holder. To view a copy of this license, visit http://creativecommons. org/licenses/by/4.0/.

(c) The Author(s) 2020 